\title{
The Proton Relay Network in the Bacterial P450s: CYP101A1 and CYP101D1
}

\author{
José A. Amaya, Dipanwita Batabyal ${ }^{\&}$ and Thomas L. Poulos* \\ Departments of Molecular Biology and Biochemistry, Pharmaceutical Sciences, and Chemistry, \\ University of California, Irvine, California 92697-3900
}

${ }^{\&}$ Current address: Amgen Inc, Higher Order Structure, Attribute Sciences, Thousand Oaks, CA 91320

*poulos@uci.edu 
Table S1: Data collection and refinement statistics

\begin{tabular}{|c|c|c|c|}
\hline & P450cam D251E-soak & P450cam D251E-co & CYP10D1 D259E \\
\hline PDB Entry & 6WFL & 6WE6 & 6WGW \\
\hline \multicolumn{4}{|l|}{ Data Collection } \\
\hline Space Group & $\mathrm{P} 22_{1} 2_{1} 2_{1}$ & $\mathrm{P} 2{ }_{1} 2_{1} 2_{1}$ & $\mathrm{P}_{4} 22$ \\
\hline Resolution $(\AA)$ & $34.25-1.6$ & $37.36-2.16$ & $48.36-1.73$ \\
\hline Radiation Source & SSRL 12-2 & ALS 5.0.2 & SSRL 12-2 \\
\hline Completeness (\%) & $98.98(98.28)$ & $98.48(90.24)$ & $99.99(99.99)$ \\
\hline No. of unique reflections & 54584 & 44051 & 139457 \\
\hline Redundancy & $2.0(2.0)$ & $2.0(1.9)$ & $2.0(2.0)$ \\
\hline $\mathrm{R}_{\text {sym }}$ or $\mathrm{R}_{\text {merge }}$ & $0.03546(0.5666)$ & $0.04673(0.429)$ & $0.02774(0.5934)$ \\
\hline $\mathrm{R}_{\text {pim }}$ & $0.03546(0.5666)$ & $0.04673(0.429)$ & $0.02774(0.5934)$ \\
\hline $\mathrm{CC}_{1 / 2}$ & $0.999(0.5666)$ & $0.995(0.609)$ & $0.999(0.453)$ \\
\hline $\mathrm{I} / \sigma(\mathrm{I})$ & $7.35(0.82)$ & $8.09(1.61)$ & $19.46(1.24)$ \\
\hline \multicolumn{4}{|l|}{ Refinement } \\
\hline Resolution $(\AA)$ & 1.6 & 1.6 & 1.73 \\
\hline B factor (mean) $\left(\AA^{2}\right)$ & 30.71 & 36.05 & 30.38 \\
\hline Rwork & 0.2195 & 0.2043 & 0.1889 \\
\hline Rfree & 0.2463 & 0.2562 & 0.2124 \\
\hline Rmsd for bonds $(\AA)$ & 0.009 & 0.009 & 0.007 \\
\hline Rmsd for angles (deg) & 1.178 & 1.06 & 0.96 \\
\hline No. of Atoms & 3551 & 6793 & 7537 \\
\hline Protein & 3229 & 6408 & 6494 \\
\hline Ligand/Ions & 56 & 110 & 141 \\
\hline Water & 266 & 275 & 902 \\
\hline
\end{tabular}


Table S2 - H-bonding distances between the critical Asp and Asp-to-Glu mutant in P450cam and CYP101D1. In CYP101D1 the homolog to P450cam Lys178 is a Gly

\begin{tabular}{|l|l|l|l|}
\hline P450cam WT & D251-R186 3.0 & D251-K178 3.0 & D251-D182 3.3 \\
\hline P450cam D251E & E251-R186 3.3 & E251-K178 3.7 & E251-D182 2.6 \\
\hline CYP101D1 WT & D259-R188 3.3 & No Lys & D259-N184 3.5 \\
\hline CYP101D1 D259E & E259-R188 2.9 & No Lys & E259-N184 3.2 \\
\hline
\end{tabular}

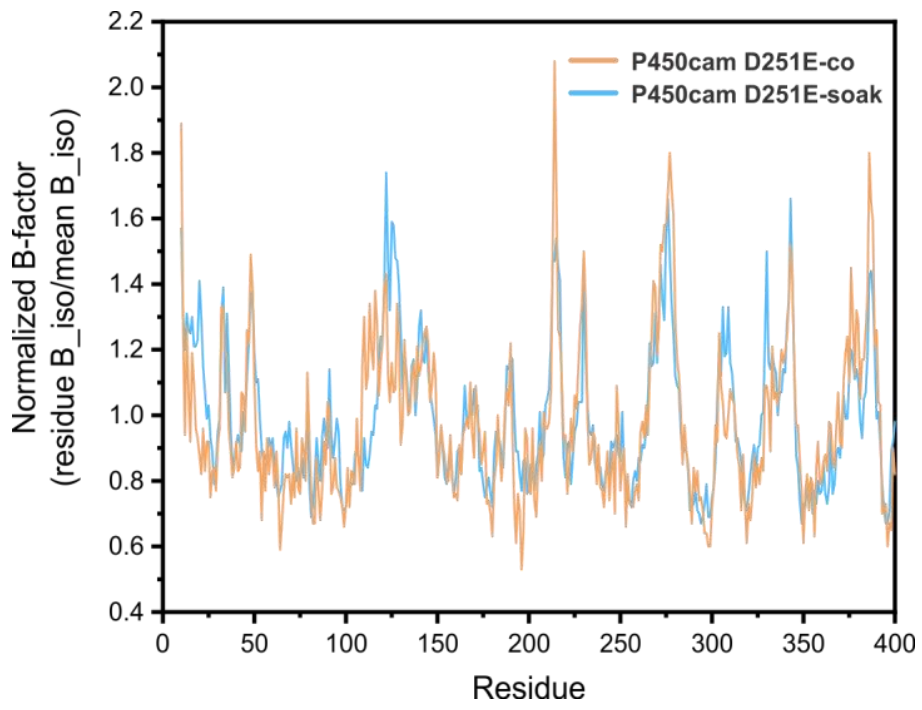

Figure S1 - Main chain normalized B-factor comparison between D251E-co (6WE6) and D251E-soak (6WFL). Increase in B-factor in D251E-soak (cyan) between residues 118-125. 

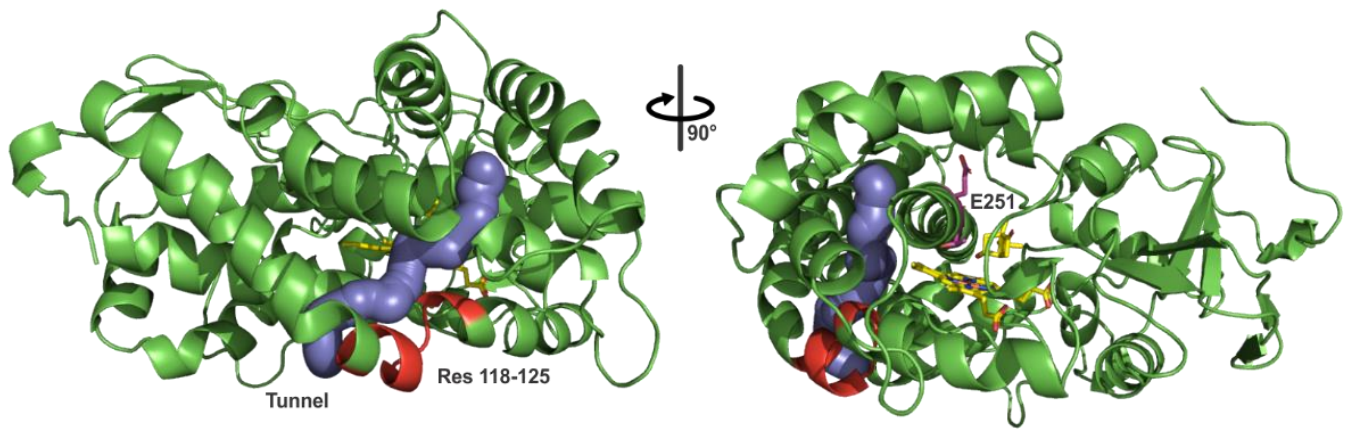

Figure S2 - P450cam D251E-soak solvent tunnel computed by Mole 2.0. Solvent tunnel is depicted in purple. Residues with higher B-factor compared to D251E-co depicted in red.

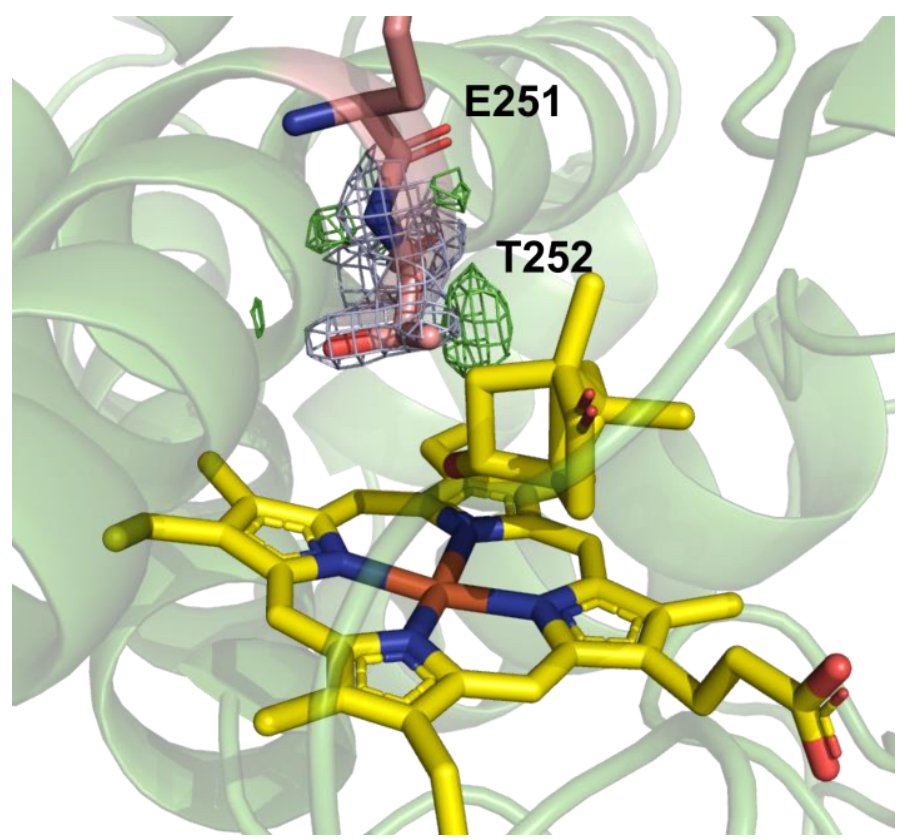

Figure S3 - Fo-Fc density around T252 in the P450cam D251E mutant suggests alternate configuration for T252 similar to the one observed in the oxy-complex. Contour was $3 \sigma$ for Fo-Fc and $2 \sigma 2 \mathrm{Fo}-\mathrm{fc}$. 
A
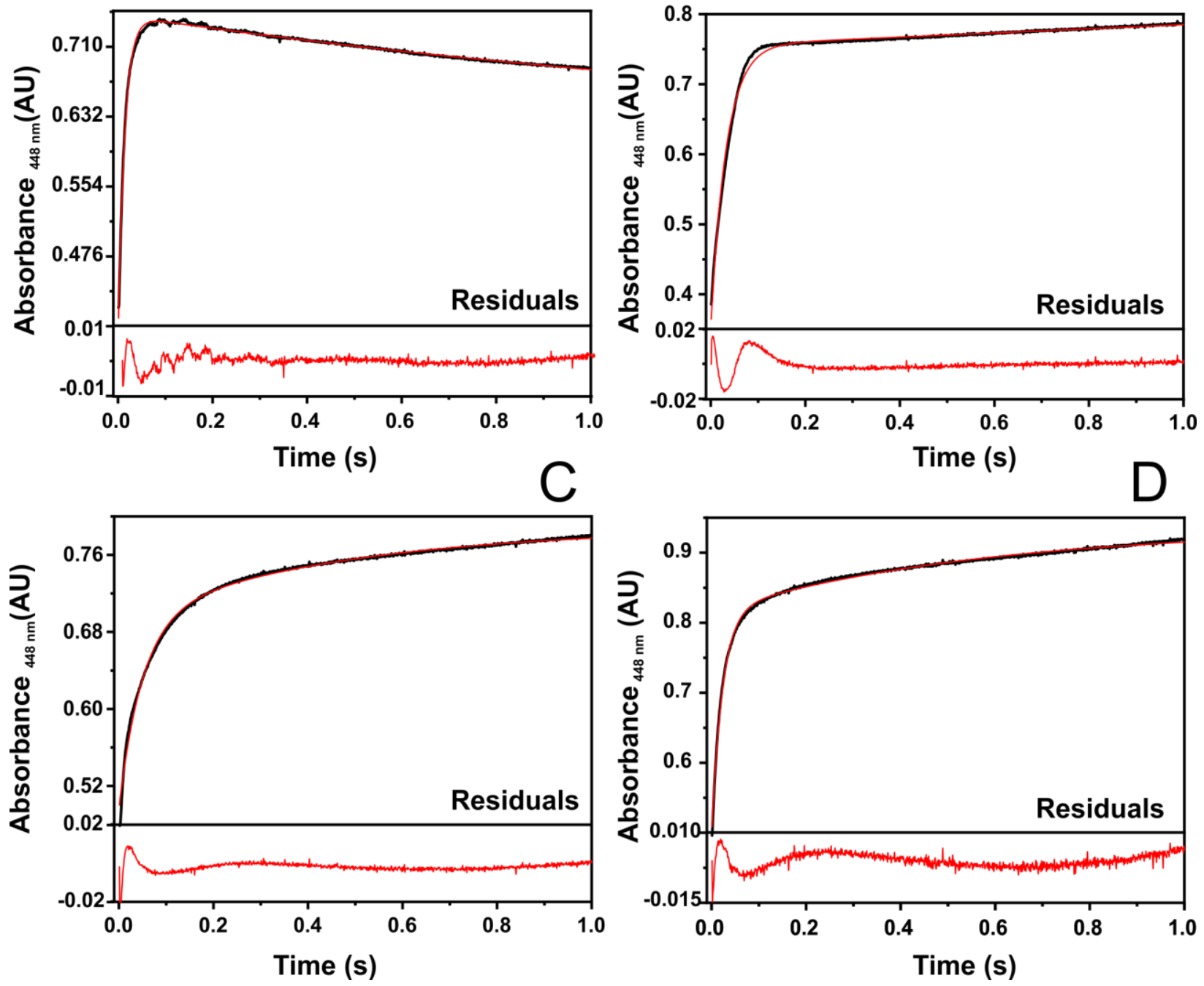

Figure S4 - First electron transfer rate representative fits. A and B represents the fit of the time-course at $448 \mathrm{~nm}$ for P450cam and P450cam D251E respectively. C and D represents the fit for the time course at $448 \mathrm{~nm}$ of CYP101D1 and CYP101D1 D259E respectively. 
A
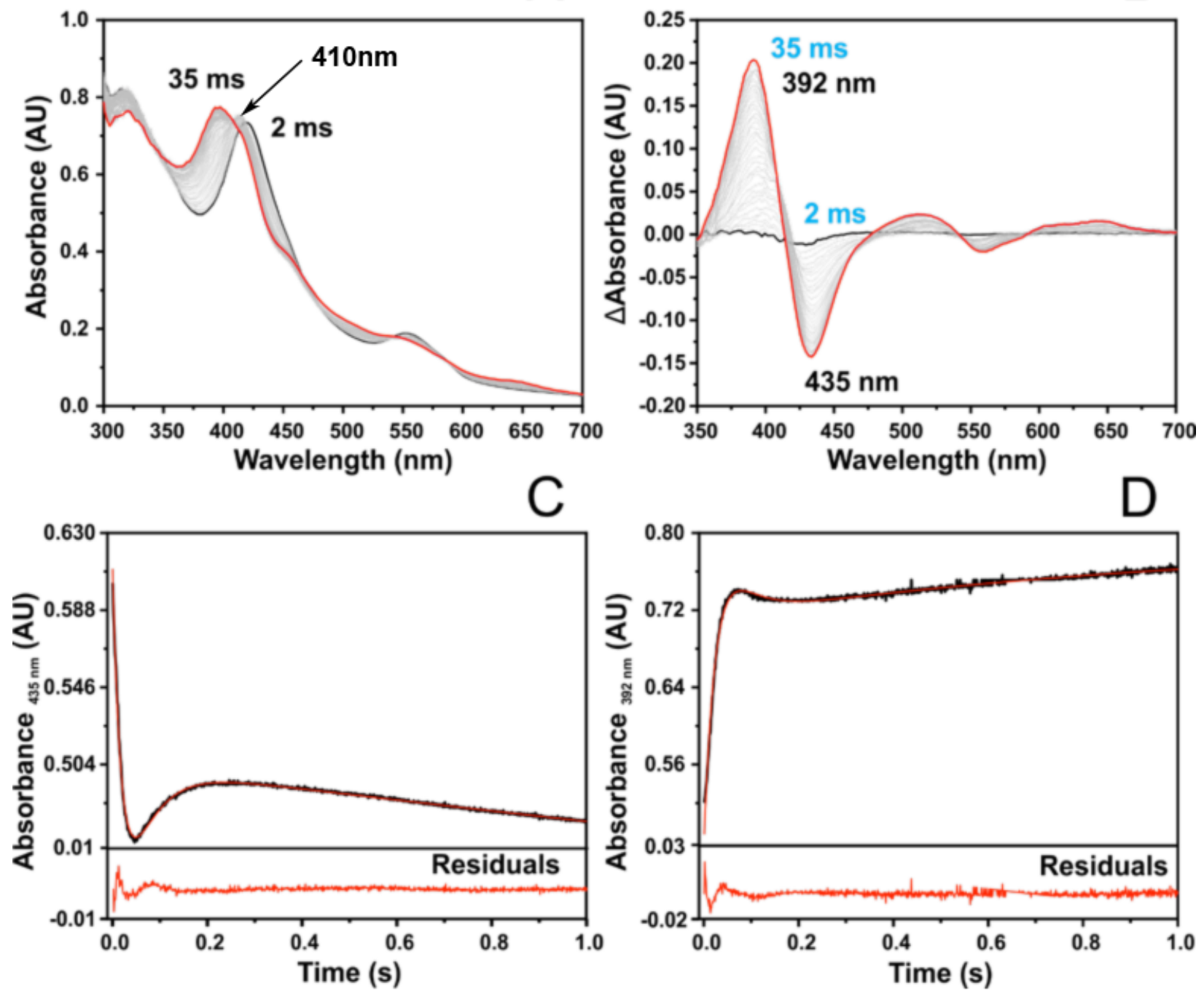

Figure S5 - Second electron transfer representative spectra and fits for P450cam. A is the raw spectra between 2-35 $\mathrm{ms}$ from the 1:1 v:v mixing of P450cam oxy-complex versus reduced ferredoxin. OxyP450cam has a Soret band maximum at 420nm which shifts to $392 \mathrm{~nm}$ after electron transfer and substrate hydroxylation. There is a build up of an intermediate at 410nm that was initially observed by Glascock et al. ${ }^{1}$ that they referred to as "perturbed" oxy-P450cam. The identity of this specieis remains to be established. B represents the difference spectrum between 2-35 ms of the same reaction. C represents the fit of the timecourse at $435 \mathrm{~nm}$ (oxy-complex). D represents the fit of the timecourse at $392 \mathrm{~nm}$ (highspin). Second electron transfer rate can be monitored by the decay of oxy-complex at $435 \mathrm{~nm}$ to high-spin at $392 \mathrm{~nm}$ since substrate binding rate is significantly faster when camphor is present at excess. 

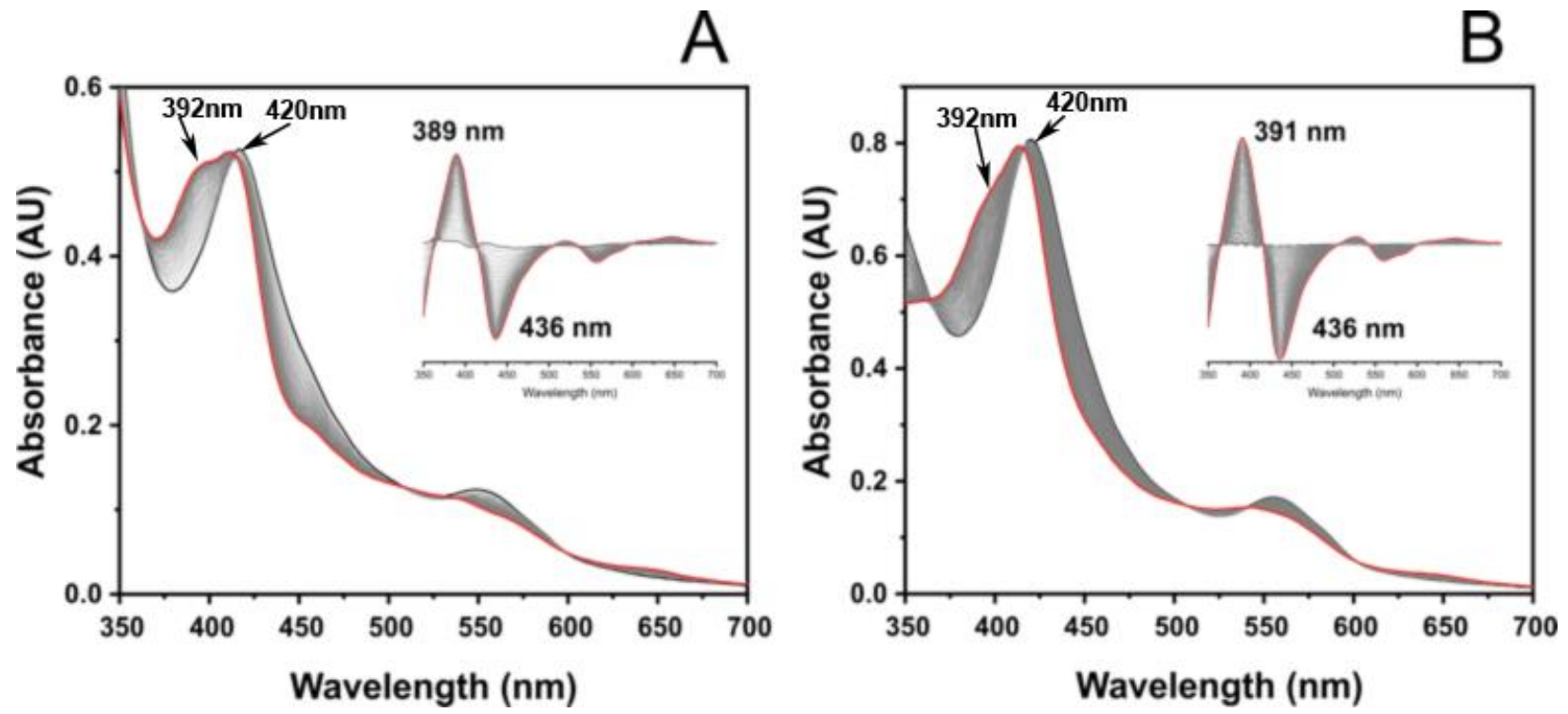

Figure S6 - Second electron transfer representative spectra for CYP101D1 and CYP101D1 D259E over the course of 1 s. A represents CYP101D1 WT and B CYP101D1 D259E. The oxy-complex Soret maximum is at $420 \mathrm{~nm}$ while $392 \mathrm{~nm}$ is the high-spin P450. Difference spectra is shown in insets. Second electron transfer rates are monitored by the decay of oxy-complex at $435 \mathrm{~nm}$ to high-spin at $392 \mathrm{~nm}$ since substrate binding rate is significantly faster when camphor is present at excess.

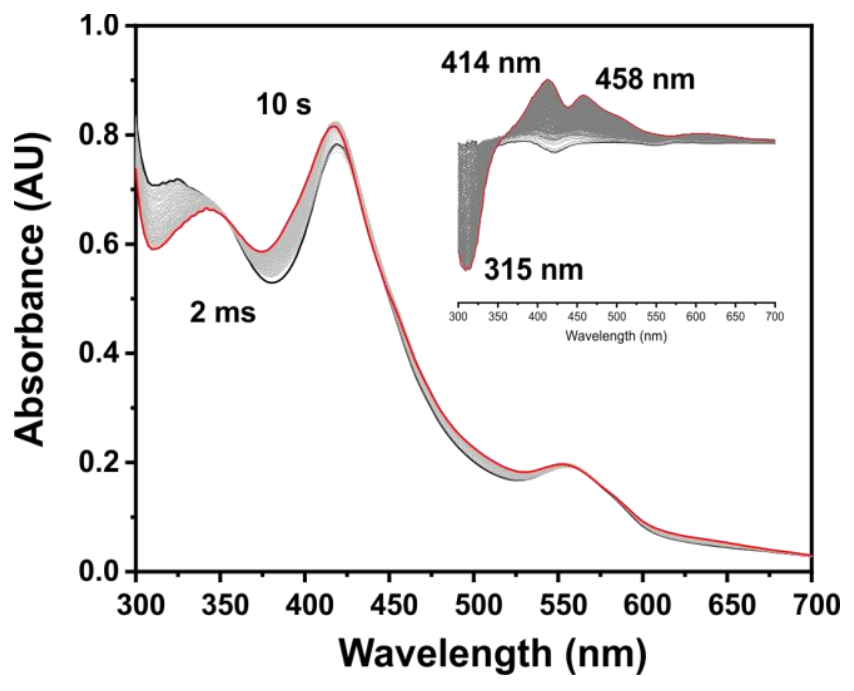

$\mathbf{F}$

Figure S7 - Stability of P450cam D251E oxy-complex in the presence of reduced PdX. The oxycomplex in the D251E mutant (Soret maximum at 420nm) is relatively stable even after $10 \mathrm{~s}$, suggesting that reduced $\mathrm{PdX}$ is not able to deliver the second electron or destabilize the oxy-complex. Inset shows the difference spectra over $10 \mathrm{~s}$. Apparent shift of the Soret band is due to oxidation of Pdx most likely from $\mathrm{O}_{2}$ leakage into the system. This is not surprising after 10 seconds. 
(1) Glascock, M. C.; Ballou, D. P.; Dawson, J. H. (2005) Direct observation of a novel perturbed oxyferrous catalytic intermediate during reduced putidaredoxin-initiated turnover of cytochrome P-450CAM: probing the effector role of putidaredoxin in catalysis. J. Biol. Chem. 280, 42134-42141. 